\title{
Increased Soluble VCAM-1 and Normal P-Selectin in Cystic Fibrosis: a Cross-Sectional Study
}

\author{
Jan K. Nowak ${ }^{1}$ - Irena Wojsyk-Banaszak ${ }^{2}$ Edyta Mądry ${ }^{3}$ - Andrzej Wykrętowicz ${ }^{4}$. \\ Patrycja Krzyżanowska ${ }^{1}$ - Sławomira Drzymała-Czyż ${ }^{1}$ - Agata Nowicka ${ }^{5}$. \\ Andrzej Pogorzelski $^{6}$ - Ewa Sapiejka ${ }^{7}$ - Wojciech Skorupa ${ }^{8} \cdot$ Mariusz Szczepanik $^{1}$. \\ Aleksandra Lisowska ${ }^{1} \cdot$ Jaroslaw Walkowiak $^{1}$
}

Received: 6 January 2017 / Accepted: 19 June 2017 / Published online: 23 June 2017

(C) The Author(s) 2017. This article is an open access publication

\begin{abstract}
Purpose As life expectancy in cystic fibrosis (CF) increases, questions regarding its potential impact on cardiovascular health arise. Soluble vascular cell adhesion molecule 1 (sVCAM-1), P-selectin (sP-selectin) are proposed as biomarkers of cardiovascular disease. We aimed to: compare their concentrations in clinically stable $\mathrm{CF}$ patients and healthy subjects (HS) and verify whether they independently correlate with $\mathrm{CF}$ characteristics.

Methods Serum sVCAM-1 and sP-selectin levels were measured using ELISA. CF was characterized using: forced expiratory volume in $1 \mathrm{~s}$, exocrine pancreatic and CF-related liver disease status, Pseudomonas aeruginosa
\end{abstract}

Jaroslaw Walkowiak

jarwalk@ump.edu.pl

1 Department of Pediatric Gastroenterology and Metabolic Diseases, Poznan University of Medical Sciences, Poznan, Poland

2 Department of Pediatric Pneumonology, Allergology and Clinical Immunology, Poznan University of Medical Sciences, Poznan, Poland

3 Department of Physiology, Poznan University of Medical Sciences, Poznan, Poland

4 Department of Cardiology-Intensive Therapy, Poznan University of Medical Sciences, Poznan, Poland

5 Department of Pulmonology, Allergology and Respiratory Oncology, Poznan University of Medical Sciences, Poznan, Poland

6 Department of Pneumology and Cystic Fibrosis, Institute of Tuberculosis and Lung Diseases, Rabka, Poland

7 Outpatient Clinic for CF Patients, Gdansk, Poland

8 Department of Lung Diseases, National Institute for Tuberculosis and Lung Diseases, Warsaw, Poland colonization, serum high-sensitivity C-reactive protein, and body mass index (BMI). CFTR genotypes were classified as severe (classes I and II) or other.

Results 108 CF patients and 51 healthy subjects volunteered for the study. In the CF group BMI was lower (median [IQR]: $20.5 \mathrm{~kg} / \mathrm{m}^{2}$ [18.4-22.2] vs. $21.6 \mathrm{~kg} / \mathrm{m}^{2}$ [19.9-23.4], $p=0.02$ ) and hsCRP levels were higher $\left(3.6 \mathrm{mg} / \mathrm{L}[1.1-7.1]\right.$ vs. $\left.0.5 \mathrm{mg} / \mathrm{dL}[0.3-1.0], p<10^{-10}\right)$. While sVCAM-1 concentrations were greater in $\mathrm{CF}$ patients $(1018 \mathrm{ng} / \mathrm{mL} \quad$ [851-1279] vs. $861 \mathrm{ng} / \mathrm{mL}$ [806-979], $\left.p<10^{-4}\right)$, sP-selectin levels did not differ $(155 \mathrm{ng} / \mathrm{mL} \quad$ [129-188] vs. $156 \mathrm{ng} / \mathrm{mL}$ [144-177], $p=0.48)$. None of the multivariable regression models was valid for the prediction of sVCAM-1 and sP-selectin in CF.

Conclusions We found higher SVCAM-1 concentrations in CF patients than in healthy subjects, which were not explained by $\mathrm{CF}$ characteristics. Further research is required to check whether SVCAM-1 is a marker of microangiopathy in $\mathrm{CF}$.

Keywords Atherosclerosis - Atherogenesis - CD62 . CD106 - GMP140 - LECAM3 - Pancreatic insufficiency

\section{Introduction}

The increasing survival of cystic fibrosis (CF) patients draws attention to CF-related cardiovascular risk. It may be expected that the CF-related chronic inflammation of the respiratory tract, diabetes, decreased antioxidant levels, altered fatty acid profile [1], gut dysbiosis, and dysregulated immune responses will promote atherogenesis. Recent studies demonstrated endothelial dysfunction and increased arterial stiffness are present in young cystic 
fibrosis patients [2, 3]. Findings from the murine model of $\mathrm{CF}$ point towards the existence of inherent causes of cardiovascular disease in $\mathrm{CF}$, which would be independent of the bronchopulmonary disease [4]. Nevertheless, still little is known about CF-related risk of cardiovascular disease and its determinants.

Among proposed biomarkers of cardiovascular risk are cell adhesion molecules-soluble vascular cell adhesion molecule 1 (sVCAM-1; CD106) and soluble P-selectin (sPselectin; CD62). VCAM-1 is crucial for leukocyte adhesion to the endothelium [5] and has a broad clinical relevance. In a group of 1246 patients with coronary artery disease followed for a mean of 2.7 years sVCAM-1 was independently associated with an increased risk of death from cardiovascular complications (2.8-fold in the upper vs. the lower quartile) and the identified effect could not be predicted by high-sensitivity C-reactive protein (hsCRP) [6]. In the Second Manifestations of ARTerial disease (SMART) study $(n=1002)$, sVCAM-1 was associated with the risk of cardiovascular events [7]. In the Bruneck study, which followed 880 persons over 20 years, sVCAM1 predicted the occurrence of atrial fibrillation after adjusting for potential confounders [8]. Plasma sVCAM-1 was shown to correlate with higher resting cerebrovascular resistance and poorer cognitive function [9].

Higher concentrations of sVCAM-1 and sP-selectin are found in the plasma of patients with peripheral artery disease than in age-matched healthy subjects [10]. de Faria et al. showed that both plasma sVCAM-1 and sP-selectin are related to greater arterial stiffness and cardiac hypertrophy. In the same study, sP-selectin indicated target organ damage even after adjustment for blood pressure [11]. Serum sVCAM-1 as well as sP-selectin are higher in metabolic syndrome patients compared to age-matched persons without it; they also positively correlate with carotid intima-media thickness [12].

A study performed in 345 healthy women revealed that sP-selectin correlates with a greater risk of cardiovascular events (2.2-fold in the upper vs. the lower quintile). The observed effect is independent of the established cardiovascular risk factors [13]. In European Prospective Investigation into Cancer and Nutrition-NL higher sP-selectin was linked to an increased risk of cardiovascular events ( $n=288)$ [7]. In persons with left ventricular ejection fraction $>50 \%$, plasma sP-selectin was shown to predict cardiac events [14]. In men at higher risk of cardiovascular disease, sP-selectin associates with the presence of carotid plaque [15]. In non-Hispanic white Americans, over a 10-year follow-up, baseline plasma sP-selectin was related to the future risk of coronary heart disease. It was also associated with greater coronary artery calcium, glycated hemoglobin [16], and-in another study-with lower ankle-brachial index [17].
Despite microvasculature dysfunction in CF [18] the available data on soluble cell adhesion molecules in CF are scarce. De Rose et al. identified increased intercellular adhesion molecule 1 (ICAM-1; CD54) and E-selectin (CD62E) concentrations in CF patients [19]. However, in the same subjects, they found normal levels of sVCAM-1. An inverse correlation between forced expiratory volume in $1 \mathrm{~s}$ (FEV1) and sP-selectin was also described [20] as well as higher sP-selectin levels in CF compared with healthy controls [21]. The above findings come from few studies with moderate sample sizes. A comprehensive analysis of potential clinical correlates of soluble cell adhesion molecule concentrations in CF is also lacking.

We hypothesized that serum sVCAM-1 and sP-selectin concentrations differ between patients with $\mathrm{CF}$ and healthy subjects (HS). The secondary hypothesis was that the clinical characteristics of CF predict the levels of the above biomarkers. Thus, we aimed to fill the gaps in our knowledge of two molecular factors potentially linked to cardiovascular health in clinically stable CF.

\section{Methods}

This study is a part of the AtheroCF project, which aims at understanding the background of atherosclerosis in CF. Patients were recruited in tertiary care centers in Poznan, Rabka, Gdansk, and Warsaw (Poland) between June 2013 and June 2016 [22]. The inclusion criteria comprised CF diagnosed according to CF Foundation Guidelines [23] and age $\geq 16$ years. Exclusion criteria for $\mathrm{CF}$ patients and $\mathrm{HS}$ were common: a family history of hypercholesterolemia and/or hypertriglyceridemia and/or cardiovascular event before 65 (women) or 55 years of age (men).

Serum hsCRP concentration was determined with immunoturbidimetry (Cobas, Roche, Rotkreuz, Switzerland). Serum soluble VCAM-1 (sVCAM-1) and sP-selectin levels were assessed using ELISA (DRG Instruments GmbH, Marburg, Germany).

All CF patients were clinically stable i.e., did not have acute exacerbation of the bronchopulmonary disease. FEV1\% values were taken from current patients' clinical records (last 6 months). CFTR mutations were divided into severe (class I or II) and other. Exocrine pancreatic status was determined using fecal elastase-1 (ELISA; Schebo Biotech, Giessen, Germany) [24, 25]. CF-associated liver disease was diagnosed according to guidelines by Debray et al. [26]. Diabetes was diagnosed by physicians specialized in diabetes care. Pseudomonas aeruginosa status was considered positive if chronic or recurrent infections were confirmed by culture [27].

The study was conceived to detect a difference equaling $1 / 2$ of standard deviation, assuming the 0.80 power of the 
test and the significance level set at 0.05 (target sample size 100 vs. 50 cases). Statistical analyses were carried out in Statistica 12 (StatSoft Inc., Tulsa, USA). The ShapiroWilk test was used to check the data for normality of the distribution and the F-test to verify whether variances were equal (not shown). Medians [1st-3rd quartiles] are reported. The Mann-Whitney $U$-test was employed to compare parameter values between groups. Spearman's rank-order correlation was calculated. Multivariable linear regression models were built in order to adjust for confounding and identify potential independent correlates of the two biomarkers (all effects). The first set of regression models included all the measured parameters with the exception of diabetes mellitus (all diabetic patients had exocrine pancreatic insufficiency); the second set investigated the following selected parameters: age, sex, FEV1\%, BMI, severe mutation, and exocrine pancreatic insufficiency.

All volunteers gave their informed written consent to participate in the study; in the case of adolescents, the informed written consent was also given by patients' parents. The study respected the revised Declaration of Helsinki and was approved by the Bioethical Committee at Poznan University of Medical Sciences (decision no. 250/10). The structure of this article is based on the Strengthening the Reporting of Observational studies in Epidemiology (STROBE) checklist [28].

\section{Results}

One hundred-eight patients with $\mathrm{CF}$ and $51 \mathrm{HS}$ were recruited for the study. The genotypes are listed in Table 1; 56 of these $(52 \%)$ contained one or two class I or II mutations and were considered to predispose to severe course of the disease. Median FEV1\% [1st-3rd quartile] was $61 \%$ [46-84\%]. Eighty-eight patients (81\%) had exocrine pancreatic insufficiency, of them, 24 had diabetes mellitus (22\%), 42 had CF-related liver disease (39\%), and 67 had positive $P$. aeruginosa status $(62 \%)$. The only missing data were two FEV1\% measurements; we did not include these cases in regression analyses (listwise deletion).

Basic characteristics and biomarker values in $\mathrm{CF}$ and $\mathrm{HS}$ are compared in Table 1. In CF higher hsCRP and sVCAM-1 concentrations were found. sVCAM-1 and sPselectin levels in CF and HS are compared in Fig. 1.

Extreme outliers that could disproportionately influence the regression models were excluded after residual analysis (one sVCAM-1 and one sP-selectin concentration). There were no significant collinearity issues. None of the models for the prediction of the two investigated biomarkers was valid. No correlations were found between FEV1\% or hsCRP and the two biomarkers. They did not correlate with each other either. In an explorative analysis, sVCAM-1 concentration was higher in $\mathrm{CF}$ patients with exocrine pancreatic insufficiency compared with those who were pancreatic-sufficient $(1043 \mathrm{ng} / \mathrm{mL}$ [897-1306] vs. $895 \mathrm{ng} / \mathrm{mL}$ [781-1007], $p=0.003)$; among pancreatic-insufficient patients there were no differences between diabetics and non-diabetics (1151 ng/ $\mathrm{mL}$ [916-1327] vs. $1026 \mathrm{ng} / \mathrm{mL}$ [899-1304], $p=0.48$ ) (Table 2).

\section{Discussion}

This study provides new data on soluble cell adhesion molecules in cystic fibrosis. While sVCAM-1 concentration was higher in $\mathrm{CF}$ than in $\mathrm{HS}$, and that of sP-selectin did not differ significantly between the two groups.

Table 1 Cystic fibrosis transmembrane conductance regulator (CFTR) genotypes of the 108 cystic fibrosis patients enrolled for the study

\begin{tabular}{ll}
\hline$n$ & Genotype \\
\hline 32 & F508del/F508del \\
15 & Unknown/unknown \\
11 & F508del/unknown \\
8 & F508del/3849+10kbC->T \\
4 & F508del/CFTRdele2,3 \\
3 & F508del/3272-26A->G \\
2 & F508del/1717-1G->A, F508del/W1282X \\
1 & F508del/2143delT, F508del/2183AA->G, F508del/2721del11, F508del/3121-2A->G, F508del/3171insC, F508del/3600+2insT, F508del/ \\
& G551D, F508del/N1303K, F508del/R117H, F508del/R352Q, F508del/R553X, F508del/R851X, CFTRdele2,3/CFTRdele2,3, \\
& CFTRdele2,3/3849+10kbC->T, CFTRdele2,3/unknown, CFTRdele2,3/N1303K, N1303K/G551D, N1303K/unknown, 1524+1G->A/ \\
& 3944delGT;406-6T->C, 1717-1G->A/unknown, 2183AA-G/1717-1G->A, 2184insA/unknown, 3272-26A > G/unknown, 3659delC/R153I, \\
& 3849+10kbC->T/3600+1G $>$ T, S1196X/Q138X, T5811/2721del11 \\
\hline
\end{tabular}


Fig. 1 Boxplots illustrating the concentrations of soluble vascular cell adhesion molecule 1 (sVCAM-1) and soluble $\mathrm{P}$-selectin (sP-selectin) in cystic fibrosis (CF) patients and healthy subjects (HS). Median values, 1 st-3rd quartiles, and 5th-95th percentiles are shown. Asterisks indicate statistical significance $\left(* * * p<10^{-4}\right)$
Table 2 Group characteristics and comparison of soluble vascular cell adhesion molecule 1 (sVCAM-1) and soluble $\mathrm{P}$-selectin (sP-selectin) in cystic fibrosis $(\mathrm{CF})$ patients and healthy subjects (HS)
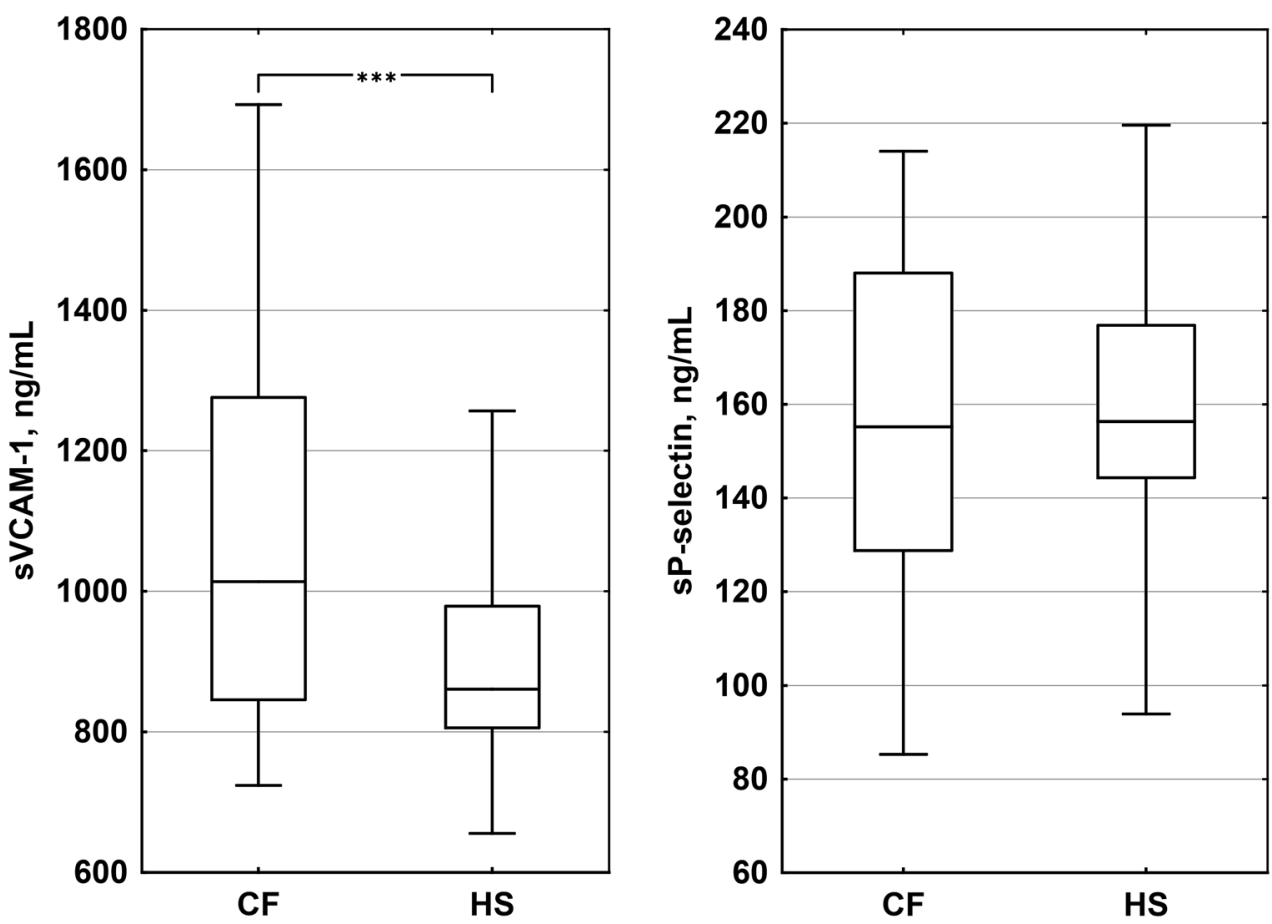

\begin{tabular}{llll}
\hline Parameter & $\mathrm{CF}, n=108$ & $\mathrm{HS}, n=51$ & $P$ \\
\hline Sex $(\mathrm{F} / \mathrm{M})$ & $61 / 47$ & $31 / 20$ & $0.73^{\mathrm{a}}$ \\
Age $($ years $)$ & $22.0(19.1-31.0)$ & $24.1(21.7-28.1)$ & 0.38 \\
Body mass index $\left(\mathrm{kg} / \mathrm{m}^{2}\right)$ & $20.5(18.4-22.2)$ & $21.6(19.9-23.4)$ & 0.02 \\
hsCRP $(\mathrm{mg} / \mathrm{L})$ & $3.6(1.1-7.1)$ & $0.5(0.3-1.0)$ & $<10^{-10}$ \\
sVCAM-1 $(\mathrm{ng} / \mathrm{mL})$ & $1018(851-1279)$ & $861(806-979)$ & $<10^{-4}$ \\
sP-selectin $(\mathrm{ng} / \mathrm{mL})$ & $155(129-188)$ & $156(144-177)$ & 0.48 \\
\hline
\end{tabular}

Median values [1st-3rd quartiles] are presented

$F$ female, $h s C R P$ high-specificity $\mathrm{C}$-reactive protein, $M$ male

${ }^{\text {a }}$ Fisher's test, two-tailed $p$ value

\section{SVCAM-1}

VCAM-1 is a ligand of very late antigen-4 (CD49d/CD29; integrin $\alpha 4 \beta 1$ ), taking part in adhesion of leukocytes to the endothelium [29]. It was found to associate with coronary artery disease [30] and its concentrations were shown to be higher in patients with hypertension [31], obesity, and diabetes [32], and also in women with preeclampsia [33]. sVCAM-1 levels were also raised in diabetic women in early pregnancy having both retino- and nephropathy compared with those who did not have retinopathy [34]. Retinopathy associated with high SVCAM- 1 concentrations in another study as well, suggesting a link not only to microangiopathy through endothelial damage, but also to neovascularization [35].

In renal-insufficient patients without diabetes and atherosclerosis, sVCAM-1 was correlated with carotid intima-media thickness [36]. In menopausal women, blood-borne microvesicle VCAM-1 was weakly associated with a positive change in carotid intima-media thickness over a period 4 years; microvesicle P-selectin was included in the principal component, predicting increases in reactive hyperemia [37]. A 6-month followup of 75 acute coronary syndrome patients demonstrated that SVCAM-1 levels predict the risk of future major cardiac events (OR 4.62; 95\% CI 1.8-11.4) [38]. High serum SVCAM-1 associates with coronary artery disease as well as lower brain-derived neurotrophic factor values during oral glucose tolerance test [39]. VCAM-1 relates to the thickness of the carotid intima-media and its plaque in rheumatoid arthritis [40]. In women, serum sVCAM-1 is inversely correlated with visceral adipose tissue [41]. In persons with hyperglycemia, moderate exercise was shown to increase sVCAM-1 [42]. 
In a murine model of reduced VCAM- 1 expression, the burden of aortic lesions was $48 \%$ lower compared with a control group [72]. In estrogen-deficient rats, sVCAM-1 decreased after augmenting the content of polyunsaturated fatty acids in the diet; this was also accompanied by reductions in both leukocyte adherence to the wall of the aorta and platelet adhesiveness [43]. VCAM-1 is overexpressed in atherotic plaque after acute hypoxia and its silencing with interfering RNA in vivo decreases granulocyte recruitment to the damaged tissue [44]. Interestingly, the association between a plasma-specific microRNA - miR-1185-and arterial stiffness was found to be partially mediated by VCAM-1 [45].

It should, however, be admitted that there is important evidence contrary to sVCAM-1 being an independent risk factor for cardiovascular events [46, 47]. Both soluble and membrane VCAM-1 are unspecific. VCAM-1 expression is raised in acute respiratory distress syndrome [48], in breast [49] and non-small cell lung cancer [50], and in rheumatoid arthritis [51], where it decreases following treatment with infliximab and methotrexate [52]. Plasma sVCAM-1 is known to respond to the exposure to air pollution with fine particulate matter $\left(\mathrm{PM}_{2.5}\right)$ [53]. The available research on the role of this molecule is mainly clinical. Overall, in patients with substantial endothelial damage sVCAM-1 may be considered a risk factor of cardiovascular disease and in other subjects an indicator of atherosclerosis progression [54].

We did not confirm the findings by De Rose et al., who found that sVCAM-1 levels did not differ in 29 patients with CF and 12 healthy volunteers [19]. In the light of the above-mentioned studies, it might be proposed that the higher sVCAM-1 concentration in $\mathrm{CF}$-as found in this study - may reflect a state of chronic inflammation, which probably predisposes to cardiovascular disease.

sVCAM-1 concentrations found by various studies differ and may not be comparable. For instance, its serum levels in HS in our study were similar to that found by some other research groups [43, 55, 56]. They are, however, higher than reported in a number of other publications $[30,33,57]$. We suppose that this variability might be due to specificity of antibodies used in ELISA kits. In fact, while the main sVCAM-1 form has the molecular weight of about $100 \mathrm{kDa}$, smaller forms also exist. Curiously, Hahne et al. showed that in mice it was 42-kDa sVCAM-1 and not 100-kDa sVCAM-1 that responded to stimulation with lipopolysaccharide [58]. Garton et al. indicated that it is the $100-\mathrm{kDa}$ sVCAM-1 that is predominant in the serum of mice and that it was cleaved from the cellular surface by ADAM (a disintegrin and metalloproteinase) metallopeptidase domain 17 (ADAM17) in response to stimulation by 12-O-tetradecanoylphorbol-13-acetate [59]. They also revealed that neutrophil elastase produces a $65-\mathrm{kDa}$
sVCAM-1 cleavage product. Singh et al. demonstrated that ADAM17-mediated sVCAM-1 shedding could be cytokine-induced [60], but did not relate to the molecular weight of obtained sVCAM-1. While some antibody suppliers state that their antibodies yield only the $100 \mathrm{kDa}$ band, we also found others that admitted that the antibodies detected unidentified $48-49-\mathrm{kDa}$ proteins. Therefore it cannot be excluded that there is a systematic bias introduced by various ELISA kits, some of which might recognize only the epitopes that are present in large, but not in smaller forms of sVCAM-1. This renders difficult the direct comparisons of sVCAM-1 concentrations obtained in different studies.

\section{sP-selectin}

P-selectin enables leukocyte adhesion to the vascular wall [61]. Its major ligands include P-selectin glycoprotein ligand-1 and TIM-1-T cell immunoglobulin and mucin domain $1[62,63]$. The role of sP-selectin in atherosclerosis development and its association with the risk of venous thromboembolism is supported by studies of SELP polymorphisms [64, 65] and research in animal models [66]. Additionally, SELP rs6128 major allele is associated with a higher sP-selectin concentration and diabetic retinopathy [67]. In dialysis patients, raised sP-selectin concentrations associate with atherosclerotic cardiovascular disease and mortality [68]. The Framingham Heart Study Offspring and Omni studies revealed correlates of sP-selectin levels: male sex, age, cigarette smoking, and other modifiable cardiovascular risk factors [69]. sP-selectin is also more abundant in the blood (serum and plasma) of patients with severe chronic venous insufficiency [70]. In community-acquired pneumonia plasma sP-selectin is increased [71] and predicts the occurrence of myocardial infarction [72]. Platelet P-selectin is associated with hypertension [73]; in patients with hypertension caused by primary aldosteronism serum, sP-selectin decreased after removal of aldosterone-secreting adrenal adenoma [74]. Plasma sP-selectin associates with waist-to-hip ratio and visceral adipose tissue in men [41]. In healthy persons, physical effort relates to lower sPselectin [75]. However, it must be underscored that there are also large cohort studies, in which plasma sP-selectin was not linked to cardiological outcomes [7, 76].

P-selectin interaction with its ligand is considered a target for novel therapies aiming at the reduction of cardiovascular risk [77]. Inclacumab, which is a monoclonal antibody against $\mathrm{P}$-selectin, protects against myocardial damage after reperfusion in non-ST-segment elevation myocardial infarction [78]. Platelet P-selectin expression is reduced by thienopyridine class inhibitors of the adenosine pyrophosphate receptor [79]. This is relevant because platelet-derived microparticles reduce the plasticity of 
FOXP3(+) regulatory $\mathrm{T}$ cells by interacting with their P-selectin $(+)$ subset, creating a pro-inflammatory milieu [80].

Serum sP-selectin levels tend to be higher than in plasma since they include P-selectin released from platelets activated during clot formation. The correlation between serum and plasma sP-selectin concentrations was shown to be linear and moderate-to-strong. Moreover, the reproducibility of measurements in both serum and plasma is excellent (intraclass correlation coefficient 0.98 and 0.92 , respectively). Serum and plasma $\mathrm{sP}$-selectin does not correlate with platelet count or mean platelet volume [81].

It seems that the role of P-selectin is dependent on where it is localized: in the serum, the platelets, or the endothelium. A cross-sectional study by Cleanthis et al. indicated that soluble-but not platelet-P-selectin correlated with spontaneous platelet aggregation in patients with intermittent claudication or stroke [82]. On the other hand, platelet-and not endothelial-P-selectin is required for the development of acute lung injury after a chemical insult: it mediates a platelet-neutrophil interaction leading to thromboxane A2 production, neutrophil adhesion, andconsequently - greater tissue damage [83].

O'Sullivan et al. found that P-selectin expression on platelets obtained in CF patients was insignificantly greater before stimulation with adenosine diphosphate, significantly higher after this stimulation at all five concentrations employed, and not entirely abolished by prostaglandin E1, which was the case in normal platelets [84]. The incomplete inhibition of platelet aggregation in CF was known previously [85]. Because similar findings were reproduced in washed platelets, O'Sullivan et al. concluded that this was due to an intrinsic platelet property. Curiously, O'Sullivan et al. did not identify CFTR or its mRNA within normal platelets, which led them to propose that the observed CF platelet hyperactivity could be traced back to megakaryocyte or related to a different chloride channel.

Sturm et al. found a higher sP-selectin concentration in $54 \mathrm{CF}$ patients-including children-compared with 55 age- and sex-matched healthy subjects [21]. In the subgroup of subjects aged 15-41 years the values compared as follows $\left(n_{\mathrm{CF}}=28 ; n_{\mathrm{HS}}=29\right): 41 \mathrm{ng} / \mathrm{mL}$ [25-56] versus $29 \mathrm{ng} / \mathrm{mL}$ [17-60]. A similar difference was observed in children aged 3-14 years. Sturm et al. considered sP-selectin to be a platelet-derived inflammatory factor. Another study, by Romano et al., reported sP-selectin to be higher in $20 \mathrm{CF}$ patients compared with $20 \mathrm{HS}$ [20] and to correlate with worse FEV1\%. In our study, sP-selectin levels in patients and HS did not differ and the medians were within the reference range established by Deneva-Koycheva et al. (102-210 ng/mL in the serum) [57].

Whereas Sturm et al. assessed the level of sP-selectin in the plasma, we measured serum P-selectin: initially present in the plasma and released from platelets on clot formation. This partly explains the higher values in our study and may hint at why Sturm et al. and Romano et al. found an effect while we did not. However, the difference between serum and plasma sP-selectin in a study by Valdes et al. was not threefold as in this case but twofold [81]. Considering that median CRP levels and FEV1\% in the group assessed by Sturm et al. and in ours were very similar, and that they also included only clinically stable patients, we expect that our assessment methods may have differed as well.

An unsurprising finding is the increased hsCRP concentration. Although the CF patients were clinically stable, it seems plausible that their hsCRP levels reflect a chronic inflammatory process in the respiratory tract. This process may be expected to negatively affect cardiac health.

The main strengths of this research include a relatively large sample size and a comprehensive characterization of CF-related clinical factors. Its main limitations comprise: the cross-sectional design, which does not permit for establishment of causation, and the investigation of biomarkers, which may give insight into pathophysiology, but cannot replace clinical endpoints. It must also be considered that the varying sensitivity of the available tests (various ELISA kits and other antibody-based techniques) lowers the utility of direct comparisons of soluble cell adhesion molecules' concentrations between studies.

\section{Conclusions}

We found higher sVCAM-1 concentrations in CF patients than in $\mathrm{HS}$, which could not be explained by CF characteristics. Further research is required to check whether sVCAM-1 is a marker of microangiopathy in CF.

Acknowledgements The authors would like to thank all the cystic fibrosis patients and healthy persons for their participation in the study.

Funding The study was supported by a grant awarded to JW by the Polish National Science Centre (DEC-2011/03/B/NZ5/0571). JKN was receiving a stipend from the Polish National Science Centre (DEC-2015/16/T/NZ5/00168).

\section{Compliance with Ethical Standards}

Conflict of interest The authors declared that they have no conflict of interest.

Ethical approval The study was approved by the Bioethical Committee at Poznan University of Medical Sciences (decision no. 250/10). All procedures performed in the study were in accordance with the Polish Medical Code of Ethics as well as with the 1964 Helsinki declaration and its later amendments. All volunteers gave their informed written consent to participate in the study; in the case of adolescents, the informed written consent was also given by patients' parents. 
Open Access This article is distributed under the terms of the Creative Commons Attribution 4.0 International License (http://crea tivecommons.org/licenses/by/4.0/), which permits unrestricted use, distribution, and reproduction in any medium, provided you give appropriate credit to the original author(s) and the source, provide a link to the Creative Commons license, and indicate if changes were made.

\section{References}

1. Drzymała-Czyż S, Krzyżanowska P, Koletzko B et al (2017) Determinants of serum glycerophospholipid fatty acids in cystic fibrosis. Int J Mol Sci. doi:10.3390/ijms18010185

2. Poore S, Berry B, Eidson D et al (2013) Evidence of vascular endothelial dysfunction in young patients with cystic fibrosis. Chest 143:939-945. doi:10.1378/chest.12-1934

3. Buehler T, Steinmann M, Singer F et al (2012) Increased arterial stiffness in children with cystic fibrosis. Eur Respir J 39:1536-1537. doi:10.1183/09031936.00212511

4. Sellers ZM, Kovacs A, Weinheimer CJ, Best PM (2013) Left ventricular and aortic dysfunction in cystic fibrosis mice. J Cyst Fibros 12:517-524. doi:10.1016/j.jcf.2012.11.012

5. Cybulsky MI, Gimbrone MA (1991) Endothelial expression of a mononuclear leukocyte adhesion molecule during atherogenesis. Science 251:788-791

6. Blankenberg S, Rupprecht HJ, Bickel C et al (2001) Circulating cell adhesion molecules and death in patients with coronary artery disease. Circulation 104:1336-1342

7. van der Leeuw J, Beulens JWJ, van Dieren S et al (2016) novel biomarkers to improve the prediction of cardiovascular event risk in type 2 diabetes mellitus. J Am Heart Assoc 5:e003048. doi:10. 1161/JAHA.115.003048

8. Willeit K, Pechlaner R, Willeit P et al (2017) Association between vascular cell adhesion molecule 1 and atrial fibrillation. JAMA Cardiol 2:516-523. doi:10.1001/jamacardio.2017.0064

9. Tchalla AE, Wellenius GA, Sorond FA et al (2017) Elevated soluble vascular cell adhesion molecule-1 is associated with cerebrovascular resistance and cognitive function. J Gerontol A Biol Sci Med Sci 72:560-566. doi:10.1093/gerona/glw099

10. Signorelli SS, Anzaldi M, Libra M et al (2016) Plasma levels of inflammatory biomarkers in peripheral arterial disease: results of a cohort study. Angiology 67:870-874. doi:10.1177/ 0003319716633339

11. de Faria AP, Ritter AMV, Sabbatini AR et al (2016) Deregulation of soluble adhesion molecules in resistant hypertension and its role in cardiovascular remodeling. Circ J 80:1196-1201. doi:10. 1253/circj.CJ-16-0058

12. Sun C-Q, Zhong C-Y, Sun W-W et al (2016) Elevated type II secretory phospholipase A2 increases the risk of early atherosclerosis in patients with newly diagnosed metabolic syndrome. Sci Rep 6:34929. doi:10.1038/srep34929

13. Ridker PM, Buring JE, Rifai N (2001) Soluble P-selectin and the risk of future cardiovascular events. Circulation 103:491-495

14. Chen W-S, Chen S-J, Lee C-C et al (2013) Plasma P-selectin predicts long-term cardiovascular events in hospitalized patients with suspected coronary artery disease and preserved left ventricular function: a 10-year follow-up study. Biomed J 36:137-143. doi:10.4103/2319-4170.113231

15. Burgess JL, Kurzius-Spencer M, Gerkin RD et al (2012) Risk factors for subclinical atherosclerosis in firefighters. J Occup Environ Med 54:328-335. doi:10.1097/JOM.0b013e318243298c

16. Bielinski SJ, Berardi C, Decker PA et al (2015) P-selectin and subclinical and clinical atherosclerosis: the multi-ethnic study of atherosclerosis (MESA). Atherosclerosis 240:3-9. doi:10.1016/j. atherosclerosis.2015.02.036
17. Berardi C, Wassel CL, Decker PA et al (2017) Elevated levels of adhesion proteins are associated with low ankle-brachial index. Angiology 68:322-329. doi:10.1177/0003319716659178

18. Rodriguez-Miguelez P, Thomas J, Seigler N et al (2016) Evidence of microvascular dysfunction in patients with cystic fibrosis. Am J Physiol Heart Circ Physiol 310:H1479-H1485. doi:10.1152/ajpheart.00136.2016

19. De Rose V, Oliva A, Messore B et al (1998) Circulating adhesion molecules in cystic fibrosis. Am J Respir Crit Care Med 157:1234-1239. doi:10.1164/ajrccm.157.4.9704134

20. Romano M, Collura M, Lapichino L et al (2001) Endothelial perturbation in cystic fibrosis. Thromb Haemost 86:1363-1367

21. Sturm A, Hebestreit H, Koenig C et al (2010) Platelet proinflammatory activity in clinically stable patients with CF starts in early childhood. J Cyst Fibros 9:179-186. doi:10.1016/j.jcf.2009. 12.004

22. Madry E, Nowak J, Wykretowicz A et al (2015) Predicting the risk of atherosclerosis in patients with cystic fibrosis-rationale and design of a prospective cohort study. J Med Sci 84:126-128

23. Foundation Cystic Fibrosis, Borowitz D, Parad RB et al (2009) Cystic Fibrosis Foundation practice guidelines for the management of infants with cystic fibrosis transmembrane conductance regulator-related metabolic syndrome during the first two years of life and beyond. J Pediatr 155:S106-S116. doi:10.1016/j.jpeds. 2009.09.003

24. Walkowiak J, Nousia-Arvanitakis S, Cade A et al (2002) Fecal elastase-1 cut-off levels in the assessment of exocrine pancreatic function in cystic fibrosis. J Cyst Fibros 1:260-264

25. Walkowiak J, Nousia-Arvanitakis S, Agguridaki C et al (2003) Longitudinal follow-up of exocrine pancreatic function in pancreatic sufficient cystic fibrosis patients using the fecal elastase-1 test. J Pediatr Gastroenterol Nutr 36:474-478

26. Debray D, Kelly D, Houwen R et al (2011) Best practice guidance for the diagnosis and management of cystic fibrosis-associated liver disease. J Cyst Fibros 10:S29-S36. doi:10.1016/ S1569-1993(11)60006-4

27. Pressler T, Bohmova C, Conway S et al (2011) Chronic Pseudomonas aeruginosa infection definition: EuroCareCF Working Group report. J Cyst Fibros 10(Suppl 2):S75-S78. doi:10.1016/ S1569-1993(11)60011-8

28. von Elm E, Altman DG, Egger M et al (2007) The Strengthening the Reporting of Observational Studies in Epidemiology (STROBE) statement: guidelines for reporting observational studies. PLoS Med 4:e296. doi:10.1371/journal.pmed.0040296

29. Kaplanski G, Marin V, Fabrigoule M et al (1998) Thrombinactivated human endothelial cells support monocyte adhesion in vitro following expression of intercellular adhesion molecule-1 (ICAM-1; CD54) and vascular cell adhesion molecule-1 (VCAM-1; CD106). Blood 92:1259-1267

30. Hulok A, Sciborski K, Marczak J et al (2014) Soluble cell adhesion molecules-does estimating sVCAM-1 and sICAM-1 concentration provide additional information about cardiovascular risk in patients with coronary artery disease? Adv Clin Exp Med 23:735-741

31. Vasilev V, Matrozova J, Elenkova A et al (2013) Asymmetric dimethylarginine (ADMA) and soluble vascular cell adhesion molecule 1(sVCAM-1) as circulating markers for endothelial dysfunction in patients with pheochromocytoma. Exp Clin Endocrinol Diabetes 121:551-555. doi:10.1055/s-0033-1353183

32. Glowinska B, Urban M, Peczynska J, Florys B (2005) Soluble adhesion molecules (sICAM-1, sVCAM-1) and selectins (sE selectin, $\mathrm{SP}$ selectin, sL selectin) levels in children and adolescents with obesity, hypertension, and diabetes. Metabolism 54:1020-1026. doi:10.1016/j.metabol.2005.03.004

33. Veas CJ, Aguilera VC, Muñoz IJ et al (2011) Fetal endothelium dysfunction is associated with circulating maternal levels of sE- 
selectin, sVCAM1, and sFlt-1 during pre-eclampsia. J Matern Fetal Neonatal Med 24:1371-1377. doi:10.3109/14767058.2011. 556204

34. Zawiejska A, Wender-Ozegowska E, Brazert J (2010) Microvascular complications are associated with low levels of maternal sE-selectin and sVCAM-1 in pregnancy complicated with pregestational diabetes mellitus. Diabetes Res Clin Pract 88:164-170. doi:10.1016/j.diabres.2010.01.011

35. Nowak M, Wielkoszyński T, Marek B et al (2008) Blood serum levels of vascular cell adhesion molecule (sVCAM-1), intercellular adhesion molecule (sICAM-1) and endothelial leucocyte adhesion molecule-1 (ELAM-1) in diabetic retinopathy. Clin Exp Med 8:159-164. doi:10.1007/s10238-008-0173-z

36. Nanayakkara PWB, Teerlink T, Stehouwer CDA et al (2005) Plasma asymmetric dimethylarginine (ADMA) concentration is independently associated with carotid intima-media thickness and plasma soluble vascular cell adhesion molecule-1 (sVCAM-1) concentration in patients with mild-to-moderate renal failure. Kidney Int 68:2230-2236. doi:10.1111/j.1523-1755.2005.00680.x

37. Miller VM, Lahr BD, Bailey KR et al (2016) Specific cellderived microvesicles: linking endothelial function to carotid artery intima-media thickness in low cardiovascular risk menopausal women. Atherosclerosis 246:21-28. doi:10.1016/j.athero sclerosis.2015.12.030

38. Postadzhiyan AS, Tzontcheva AV, Kehayov I, Finkov B (2008) Circulating soluble adhesion molecules ICAM-1 and VCAM-1 and their association with clinical outcome, troponin $\mathrm{T}$ and C-reactive protein in patients with acute coronary syndromes. Clin Biochem 41:126-133. doi:10.1016/j.clinbiochem.2007.09. 001

39. Lee I-T, Wang J-S, Lee W-J et al (2017) The synergistic effect of vascular cell adhesion molecule-1 and coronary artery disease on brain-derived neurotrophic factor. Clin Chim Acta 466:194-200. doi:10.1016/j.cca.2017.01.026

40. Dessein PH, Joffe BI, Singh S (2005) Biomarkers of endothelial dysfunction, cardiovascular risk factors and atherosclerosis in rheumatoid arthritis. Arthritis Res Ther 7:R634-R643. doi:10. 1186/ar1717

41. Christoph MJ, Allison MA, Pankow JS et al (2016) Impact of adiposity on cellular adhesion: the Multi-Ethnic Study of Atherosclerosis (MESA). Obesity (Silver Spring) 24:223-230. doi: 10.1002/oby.21245

42. Nygaard H, Falch GS, Whist JE et al (2017) Acute effects of post-absorptive and postprandial moderate exercise on markers of inflammation in hyperglycemic individuals. Eur J Appl Physiol 117:787-794. doi:10.1007/s00421-017-3576-2

43. Haliga RE, Iancu RI, Butcovan D, Mocanu V (2013) Flaxseed prevents leukocyte and platelet adhesion to endothelial cells in experimental atherosclerosis by reducing SVCAM-1 and vWF. ScientificWorldJournal 2013:303950. doi:10.1155/2013/303950

44. Sager HB, Dutta P, Dahlman JE et al (2016) RNAi targeting multiple cell adhesion molecules reduces immune cell recruitment and vascular inflammation after myocardial infarction. Sci Transl Med 8:342ra80. doi:10.1126/scitranslmed.aaf1435

45. Deng H, Song Z, Xu H et al (2017) MicroRNA-1185 promotes arterial stiffness though modulating VCAM-1 and E-selectin expression. Cell Physiol Biochem 41:2183-2193. doi:10.1159/ 000475576

46. Luc G, Arveiler D, Evans A et al (2003) Circulating soluble adhesion molecules ICAM-1 and VCAM-1 and incident coronary heart disease: the PRIME Study. Atherosclerosis 170:169-176

47. Kunutsor SK, Bakker SJL, Dullaart RPF (2017) Soluble vascular cell adhesion molecules may be protective of future cardiovascular disease risk: findings from the PREVEND prospective cohort study. J Atheroscler Thromb. doi:10.5551/jat.38836
48. Attia EF, Jolley SE, Crothers K et al (2016) Soluble vascular cell adhesion molecule-1 (sVCAM-1) is elevated in bronchoalveolar lavage fluid of patients with acute respiratory distress syndrome. PLoS ONE 11:e0149687. doi:10.1371/journal.pone.0149687

49. Thielemann A, Baszczuk A, Kopczyński Z et al (2014) The clinical usefulness of assessing the concentration of cell adhesion molecules sVCAM-1 and sICAM-1 in the serum of women with primary breast cancer. Contemp Oncol (Pozn) 18:252-259. doi:10.5114/wo.2014.43492

50. Zhou F, Chen J, Tao G et al (2014) Increased levels of exhaled sICAM1, sVCAM1, and sE-selectin in patients with non-small cell lung cancer. Respir Med 108:1670-1676. doi:10.1016/j. rmed.2014.08.003

51. Kolopp-Sarda MN, Guillemin F, Chary-Valckenaere I et al (2001) Longitudinal study of rheumatoid arthritis patients discloses sustained elevated serum levels of soluble CD106 (VCAM). Clin Exp Rheumatol 19:165-170

52. Klimiuk PA, Sierakowski S, Domysławska I et al (2004) Reduction of soluble adhesion molecules (sICAM-1, sVCAM-1, and sE-selectin) and vascular endothelial growth factor levels in serum of rheumatoid arthritis patients following multiple intravenous infusions of infliximab. Arch Immunol Ther Exp (Warsz) 52:36-42

53. Pope CA, Bhatnagar A, McCracken JP et al (2016) Exposure to fine particulate air pollution is associated with endothelial injury and systemic inflammation. Circ Res 119:1204-1214. doi:10. 1161/CIRCRESAHA.116.309279

54. George SJ (2010) Atherosclerosis: molecular and cellular mechanisms. Wiley, Weinheim

55. Nash MC, Wade AM, Shah V, Dillon MJ (1996) Normal levels of soluble E-selectin, soluble intercellular adhesion molecule-1 (sICAM-1), and soluble vascular cell adhesion molecule-1 (sVCAM-1) decrease with age. Clin Exp Immunol 103:167-170. doi:10.1046/j.1365-2249.1996.925616.x

56. Uğurlu N, Gerceker S, Yülek F et al (2013) The levels of the circulating cellular adhesion molecules ICAM-1, VCAM-1 and endothelin-1 and the flow-mediated vasodilatation values in patients with type 1 diabetes mellitus with early-stage diabetic retinopathy. Intern Med 52:2173-2178

57. Deneva-Koycheva TI, Vladimirova-Kitova LG, Angelova EA, Tsvetkova TZ (2011) Serum levels of siCAM-1, sVCAM-1, sEselectin, sP-selectin in healthy Bulgarian people. Folia Med (Plovdiv) 53:22-28

58. Hahne M, Lenter M, Jäger U, Vestweber D (1994) A novel soluble form of mouse VCAM-1 is generated from a glycolipidanchored splicing variant. Eur J Immunol 24:421-428. doi:10. 1002/eji.1830240223

59. Garton KJ, Gough PJ, Philalay J et al (2003) Stimulated shedding of vascular cell adhesion molecule 1 (VCAM-1) is mediated by tumor necrosis factor-alpha-converting enzyme (ADAM 17). J Biol Chem 278:37459-37464. doi:10.1074/jbc.M305877200

60. Singh RJR, Mason JC, Lidington EA et al (2005) Cytokine stimulated vascular cell adhesion molecule-1 (VCAM-1) ectodomain release is regulated by TIMP-3. Cardiovasc Res 67:39-49. doi:10.1016/j.cardiores.2005.02.020

61. Woollard KJ, Suhartoyo A, Harris EE et al (2008) Pathophysiological levels of soluble P-selectin mediate adhesion of leukocytes to the endothelium through Mac-1 activation. Circ Res 103:1128-1138. doi:10.1161/CIRCRESAHA.108.180273

62. Roller J, Wang Y, Rahman M et al (2013) Direct in vivo observations of P-selectin glycoprotein ligand-1-mediated leukocyte-endothelial cell interactions in the pulmonary microvasculature in abdominal sepsis in mice. Inflamm Res 62:275-282. doi:10.1007/s00011-012-0575-y

63. Angiari S, Donnarumma T, Rossi B et al (2014) TIM-1 glycoprotein binds the adhesion receptor P-selectin and mediates $\mathrm{T}$ cell 
trafficking during inflammation and autoimmunity. Immunity 40:542-553. doi:10.1016/j.immuni.2014.03.004

64. Reiner AP, Carlson CS, Thyagarajan B et al (2008) Soluble P-selectin, SELP polymorphisms, and atherosclerotic risk in European-American and African-African young adults: the Coronary Artery Risk Development in Young Adults (CARDIA) Study. Arterioscler Thromb Vasc Biol 28:1549-1555. doi:10. 1161/ATVBAHA.108.169532

65. Ay C, Jungbauer LV, Kaider A et al (2008) P-selectin gene haplotypes modulate soluble P-selectin concentrations and contribute to the risk of venous thromboembolism. Thromb Haemost 99:899-904. doi:10.1160/TH07-11-0672

66. Woollard KJ, Lumsden NG, Andrews KL et al (2014) Raised soluble P-selectin moderately accelerates atherosclerotic plaque progression. PLoS ONE 9:e97422. doi:10.1371/journal.pone. 0097422

67. Penman A, Hoadley S, Wilson JG et al (2015) P-selectin plasma levels and genetic variant associated with diabetic retinopathy in African Americans. Am J Ophthalmol 159(1152-1160):e2. doi:10.1016/j.ajo.2015.03.008

68. Scialla JJ, Plantinga LC, Kao WHL et al (2011) Soluble P-selectin levels are associated with cardiovascular mortality and sudden cardiac death in male dialysis patients. Am J Nephrol 33:224-230. doi: $10.1159 / 000324517$

69. Lee DS, Larson MG, Lunetta KL et al (2008) Clinical and genetic correlates of soluble P-selectin in the community. J Thromb Haemost 6:20-31. doi:10.1111/j.1538-7836.2007.02805.x

70. Bryan LJ, Callas PW, Criqui MH, Cushman M (2012) Higher soluble P-selectin is associated with chronic venous insufficiency: the San Diego Population Study. Thromb Res 130:716-719. doi:10.1016/j.thromres.2012.07.012

71. Cangemi R, Pignatelli P, Carnevale R et al (2016) Low-grade endotoxemia, gut permeability and platelet activation in community-acquired pneumonia. J Infect 73:107-114. doi:10.1016/j. jinf.2016.05.013

72. Cangemi R, Casciaro M, Rossi E et al (2014) Platelet activation is associated with myocardial infarction in patients with Pneumonia. J Am Coll Cardiol 64:1917-1925. doi:10.1016/j.jacc.2014. 07.985

73. Preston RA, Coffey JO, Materson BJ et al (2007) Elevated platelet P-selectin expression and platelet activation in high risk patients with uncontrolled severe hypertension. Atherosclerosis 192:148-154. doi:10.1016/j.atherosclerosis.2006.04.028

74. Petramala L, Iacobellis G, Carnevale R et al (2016) Enhanced soluble serum CD40L and serum p-selectin levels in primary aldosteronism. Horm Metab Res 48:440-445. doi:10.1055/s0042-103588

75. Jerzykowska S, Cymerys M, Roszak M et al (2014) The analysis of the influence of short term physical effort on selected endothelial function parameters and blood vessels stiffness features among young and healthy men. Pol Merkur Lek 36:373-378

76. Schnabel RB, Yin X, Larson MG et al (2013) Multiple inflammatory biomarkers in relation to cardiovascular events and mortality in the community. Arterioscler Thromb Vasc Biol 33:1728-1733

77. Patel MS, Miranda-Nieves D, Chen J et al (2017) Targeting $\mathrm{P}$-selectin glycoprotein ligand-1/P-selectin interactions as a novel therapy for metabolic syndrome. Transl Res 183:1-13. doi:10. 1016/j.trsl.2016.11.007

78. Stähli BE, Gebhard C, Duchatelle V et al (2016) Effects of the P-selectin antagonist inclacumab on myocardial damage after percutaneous coronary intervention according to timing of infusion: insights from the SELECT-ACS trial. J Am Heart Assoc. doi:10.1161/JAHA.116.004255

79. Wagner H, Lood C, Borna $\mathrm{C}$ et al (2016) Prasugrel $5 \mathrm{mg}$ inhibits platelet P-selectin and GPIIb-IIIa expression in very elderly and non elderly: results from the GENERATIONS trial, a pharmacodynamic study in stable CAD patients. J Thromb Thrombolysis 42:369-375. doi:10.1007/s11239-016-1372-1

80. Dinkla S, van Cranenbroek B, van der Heijden WA et al (2016) Platelet microparticles inhibit IL-17 production by regulatory $\mathrm{T}$ cells through P-selectin. Blood 127:1976-1986. doi:10.1182/ blood-2015-04-640300

81. Valdes V, Nardi MA, Elbaum L, Berger JS (2015) Reproducibility over time and effect of low-dose aspirin on soluble $\mathrm{P}$-selectin and soluble CD40 ligand. J Thromb Thrombolysis 40:83-87. doi:10.1007/s11239-015-1179-5

82. Cleanthis M, Smout J, Bhattacharya V et al (2008) Soluble but not platelet P-selectin correlates with spontaneous platelet aggregation: a pilot study. Clin Appl Thromb 14:227-233. doi: $10.1177 / 1076029607305915$

83. Zarbock A, Singbartl K, Ley K (2006) Complete reversal of acidinduced acute lung injury by blocking of platelet-neutrophil aggregation. J Clin Invest 116:3211-3219. doi:10.1172/JCI29499

84. O'Sullivan BP (2005) Platelet activation in cystic fibrosis. Blood 105:4635-4641. doi:10.1182/blood-2004-06-2098

85. Samuels CE, Robinson PG, Elliott RB (1975) Decreased inhibition of platelet aggregation by PGE1 in children with cystic fibrosis and their parents. Prostaglandins 10:617-621 